PROCEEDINGS OF THE

AMERICAN MATHEMATICAL SOCIETY

Volume 126, Number 5, May 1998, Pages 1355-1361

S $0002-9939(98) 04188-4$

\title{
THE POINT SPECTRUM OF FROBENIUS-PERRON AND KOOPMAN OPERATORS
}

\author{
J. DING
}

(Communicated by Palle E. T. Jorgensen)

\begin{abstract}
We present some results on the point spectrum of the FrobeniusPerron operator $P: L^{1} \rightarrow L^{1}$ and the Koopman operator $U: L^{\infty} \rightarrow L^{\infty}$ associated with a nonsingular transformation $S: X \rightarrow X$ on a $\sigma$-finite measure space $(X, \Sigma, \mu)$.
\end{abstract}

\section{INTRODUCTION}

Let $(X, \Sigma, \mu)$ be a complete $\sigma$-finite measure space, let $L^{1} \equiv L^{1}(\Sigma)$ be the Banach space of all $\mu$-integrable complex functions defined on $X$ with the $L^{1}$-norm $\|f\|_{1}=\int|f| d \mu$, and let $L^{\infty} \equiv L^{\infty}(X)$ be the Banach space of all bounded almost everywhere complex $\Sigma$-measurable functions on $X$ with the $L^{\infty}$-norm $\|g\|_{\infty}=$ ess $\sup |g|$.

Let a $\Sigma$-measurable transformation $S: X \rightarrow X$ be nonsingular, i.e., $\left(\mu \circ S^{-1}\right)(A)$ $\equiv\left(S^{-1}(A)\right)=0$ for all $A \in \Sigma$ such that $\mu(A)=0$. The operator $P: L^{1} \rightarrow L^{1}$ defined by

$$
\int_{A} \operatorname{Pfd} \mu=\int_{S^{-1}(A)} f d \mu, \quad A \in \Sigma,
$$

and the operator $U: L^{\infty} \rightarrow L^{\infty}$ defined by $U g=g \circ S$ are called the FrobeniusPerron operator and the Koopman operator associated with $S$, respectively. $\|P\|_{1}=$ $\|U\|_{\infty}=1$, and $U$ is the dual of $P$ in the sense that $\int(P f) g d \mu=\int f U g d \mu$ for $f \in L^{1}$ and $g \in L^{\infty}$.

A positive weak contraction $P: L^{1} \rightarrow L^{1}$ is often called a Markov process. The Frobenius-Perron operator is not only a Markov process, but also keeps the norm of nonnegative functions from its definition. An excellent book on the ergodic theory of Markov processes is [12], in which the asymptotic behavior of the iterates of the process was studied. However, the spectrum including the point spectrum of Markov processes has not been explored.

The motivation for studying Frobenius-Perron operators and Koopman operators associated with nonsingular transformations is to investigate the asymptotic behavior of a chaotic deterministic dynamical system from the statistical point of

Received by the editors October 23, 1995 and, in revised form, August 29, 1996, September 23, 1996 and October 14, 1996.

1991 Mathematics Subject Classification. Primary 47A35, 28D05, 47A10, 47B38.

Key words and phrases. Frobenius-Perron operator, Koopman operator.

Research was supported in part by a grant from the Minority Scholars Program through the University of Southern Mississippi. 
view. It is well-known [14] that $f \in L^{1}$ is a fixed point of $P$ if and only if the complex measure $\mu_{f}$ defined by

$$
\mu_{f}(A)=\int_{A} f d \mu, \quad A \in \Sigma,
$$

which is absolutely continuous with respect to $\mu$, is invariant under $S$, that is, $\mu_{f}\left(S^{-1}(A)\right)=\mu_{f}(A)$ for $A \in \Sigma$. For the importance of this problem and many applications, see the monographs [2], [14].

Recently, motivated by the convergence rate analysis (see [3], [6], [8], [13]) of Ulam-type numerical methods ([18], [15]) for computing fixed points of $P$ based on spectral approximation techniques and the Cauchy integral of operators [9], some general properties and a partial spectral analysis of Frobenius-Perron operators and Koopman operators have been given in [4], [5], [7]. In this paper we further study the point spectrum problem of such operators, based on the decomposition theorems for Frobenius-Perron operators [7] and for Koopman operators [4]. Our results in the next two sections are new, and results in Section 3 will extend the known ones for measure-preserving transformations to more general nonsingular ones.

The spectral theory for Frobenius-Perron operators and Koopman operators gives various generalizations of the Frobenius-Perron theory for nonnegative matrices to the infinite dimensional case. In [1], the cyclic structure of eigenvalues of irreducible nonnegative matrices has been extended to the case of positive linear maps of von Neumann algebra semigroups of such maps. Spectral properties of irreducible positive linear maps of a finite-dimensional $C^{*}$-algebra into itself have been described in [10], in which a version of the Frobenius-Perron theorem was given. An ergodic decomposition theory for $C^{*}$-finitely correlated translation invariant states on a quantum spin chain has been developed in [11], giving the group structure of the peripheral point spectrum of some ergodic positive linear map. From Theorem V.4.4 in [16], the spectrum of any Koopman operator, and thus the spectrum of any Frobenius-Perron operator, is a cyclic subset of the closed unit disk. Hence the peripheral spectrum has a group structure. While this has not been proved for the peripheral point spectrum for Frobenius-Perron operators, the peripheral point spectrum of Koopman operators associated with ergodic onto transformations does have the group structure, which generalizes known results for measure-preserving transformations (see Section 3).

General properties of eigenfunctions and eigenvalues of ergodic measure-preserving transformations have been studied based on the spectral property of unitary operators on a Hilbert space (see, for example, [17], [19]). However, the eigenproblem for general Frobenius-Perron operators and Koopman operators has not been seriously investigated to the knowledge of the author. $S: X \rightarrow X$ preserves $\mu$ if and only if the constant function $f(x) \equiv 1$ is a fixed point of the corresponding Frobenius-Perron operator $P: L^{1} \rightarrow L^{1}$. The spectrum problem for measure-preserving transformations plays a key role in the classification of dynamical systems, and by definition it is defined as the spectrum of the restriction of the Koopman operator, $\left.U\right|_{L^{2}}$, which is an isometric (unitary) operator for the (invertible) measure-preserving transformation. The spectrum problem for Koopman operators is more difficult, since its domain is $L^{\infty}$, and only when $S$ is onto, $U$ is isometric (see Theorem 2.1 below). The spectrum problem of general FrobeniusPerron operators is also difficult. In fact, it is still an open problem, and so is the 
spectrum of general Markov processes. In this paper we give a partial solution to this difficult problem, and the introduction of the nested sequence of sub- $\sigma$-algebras $\left\{S^{-k} \Sigma\right\}$ in the next section seems a right approach toward proving a conjecture in [5] on the spectrum of general Frobenius-Perron operators.

\section{The point SPECTRUm of $P$}

Throughout the paper we assume that the measure space $\left(X, S^{-1} \Sigma, \mu\right)$ is also $\sigma$-finite, where $S^{-1} \Sigma=\left\{S^{-1}(A): A \in \Sigma\right\}$. Then the Radon-Nikodym derivative $h \equiv d \mu \circ S^{-1} / d \mu$ is finite valued $\mu$-a.e. Let the support of $h$ be $\operatorname{supp} h=\{x \in X$ : $h(x) \neq 0\}$. Denote by $D$ and $\partial D$ the closed unit disk and the unit circle of the complex plane, respectively.

The analysis of the point spectrum of $P$ and $U$ is based on the following result whose proof can be found in [4], [5], and [7].

Theorem 2.1. (i) $L^{1}$ is the topological sum of $R(E)=L^{1}\left(S^{-1} \Sigma\right)$ which is the range of the conditional expectation $E: L^{1} \rightarrow L^{1}$ corresponding to the sub- $\sigma$-algebra $S^{-1} \Sigma$ and the null space $N(P)$ of $P$. Moreover, $N(P)=N(E)$, and $P$ is isometric on $R(E)$.

(ii) $L^{\infty}$ is the topological sum of $N(U)=L^{\infty}\left((\operatorname{supp} h)^{c}\right)$ and $L^{\infty}(\operatorname{supp} h)$, and $U$ is isometric on $L^{\infty}(\operatorname{supp} h)$.

(iii) The following conditions are equivalent: (a) $P$ is injective; (b) $U$ is surjective; (c) $S^{-1} \Sigma=\Sigma$; (d) $E: L^{1} \rightarrow L^{1}$ is the identity operator.

(iv) The following conditions are equivalent: (a) $U$ is injective; (b) $P$ is surjective; (c) $\mu \circ S^{-1} \cong \mu$; (d) $\operatorname{supp} h=X$.

(v) If $P$ is injective, then $\sigma(P)$, the spectrum of $P$, is either $D$ or a cyclic subset of $\partial D$, depending on whether $0 \in \sigma(P)$ or not.

(vi) If $U$ is injective, then $\sigma(U)$ is either $D$ or a cyclic subset of $\partial D$, depending on whether $0 \in \sigma(U)$ or not.

Now we begin to investigate the point spectrum of $P$ and $U$. First we have the following lemma which was also given in [3].

Proposition 2.1. If $P f=\lambda f$ with $\lambda \neq 1$, then $\int f d \mu=0$.

Proof. By the definition of $P$,

$$
\int f d \mu=\int P f d \mu=\lambda \int f d \mu .
$$

Since $\lambda \neq 1$, the conclusion follows.

The next result shows that for an injective $P$, there is no eigenvalue $\lambda$ with $|\lambda|<1$. Let $\sigma_{p}(P) \subset \sigma(P)$ be the point spectrum of $P$.

Proposition 2.2. If $P$ is injective, then $\sigma_{p}(P) \subset \partial D$.

Proof. $P f=\lambda f$. By Theorem 2.1(i), $P$ is isometric. Hence

$$
0=\|P f-\lambda f\|_{1} \geq\|P f\|_{1}-|\lambda|\|f\|_{1}=(1-|\lambda|)\|f\|_{1} .
$$

Now $f \neq 0$ and $|\lambda| \leq 1$ imply that $|\lambda|=1$.

From Theorem 2.1(iii), the condition of the above proposition is equivalent to $S^{-1} \Sigma=\Sigma$. Now we consider a possible generalization. For $k=0,1, \ldots$, let $\Sigma_{k}=S^{-k} \Sigma$. Then $S^{-1} \Sigma_{k}=\Sigma_{k+1}$ and $\left\{\Sigma_{k}\right\}_{k=0}^{\infty}$ is a decreasing sequence of sub- $\sigma$ algebras of $\Sigma$. Either $\Sigma_{k+1} \neq \Sigma_{k}$ for all $k$ or $\Sigma_{k+1}=\Sigma_{k}$ for some $k$ which implies 
that $\Sigma_{i+1}=\Sigma_{i}$ for all $i \geq k$. Denote $\Sigma_{\infty}=\bigcap_{k=0}^{\infty} \Sigma_{k}$. Note that if $\left(X, \Sigma_{k}, \mu\right)$ is $\sigma$-finite, so is $\left(X, \Sigma_{i}, \mu\right)$ for any $i<k$.

Let $0 \leq k \leq \infty$. Since $S$ is nonsingular with respect to $\Sigma$, so is $S$ with respect to $\Sigma_{k}$. Thus the corresponding Frobenius-Perron operator

$$
P_{k}: L^{1}\left(\Sigma_{k}\right) \rightarrow L^{1}\left(\Sigma_{k}\right)
$$

is well-defined. Sometimes we denote $P=P_{0}$ for convenience. If $\left(X, \Sigma_{k}, \mu\right)$ is $\sigma$ finite, and if we let $E_{k}: L^{1} \rightarrow L^{1}$ be the conditional expectation operator (see, for example, [7]) corresponding to $\Sigma_{k}$, then $P_{k}=E_{k} P_{i}$ on $L^{1}\left(\Sigma_{k}\right)$ for $i=0,1, \ldots, k$ since for any $f \in L^{1}\left(\Sigma_{k}\right)$ and $A \in \Sigma_{k}$,

$$
\int_{A} P_{k} f d \mu=\int_{S^{-1}(A)} f d \mu=\int_{A} P_{i} f d \mu=\int_{A} E_{k} P_{i} f d \mu .
$$

Since $E_{k}$ is identity on $L^{1}\left(\Sigma_{k}\right)$, it follows that

$$
P_{k} E_{k}=E_{k} P_{i} E_{k}, \quad i=0,1, \ldots, k .
$$

Furthermore, by Theorem 2.1(i), if $\left(X, \Sigma_{k+1}, \mu\right)$ is $\sigma$-finite, then $L^{1}\left(\Sigma_{k}\right)$ is the topological sum of $R\left(E_{k+1}\right)=L^{1}\left(\Sigma_{k+1}\right)$ and $N\left(\left.E_{k+1}\right|_{L^{1}\left(\Sigma_{k}\right)}\right)=N\left(P_{k}\right)$ and $P_{k}$ is isometric on $L^{1}\left(\Sigma_{k+1}\right)$. If $\Sigma_{k+1}=\Sigma_{k}$ and $\left(X, \Sigma_{k}, \mu\right)$ is $\sigma$-finite for some $k<\infty$, by Theorem 2.1(iii), $P_{k}$ is injective, hence $\sigma_{p}\left(P_{k}\right) \subset \partial D$.

Proposition 2.3. If $\Sigma_{k+1}=\Sigma_{k}$ for some $k<\infty$ and $\left(X, \Sigma_{k}, \mu\right)$ is $\sigma$-finite, then $\sigma_{p}(P) \subset \partial D \cup\{0\}$.

Proof. Suppose $P f=\lambda f$ for some $\lambda \neq 0$ and $f \neq 0$. Let $f=f_{1}+f^{1}$ with $f_{1} \in L^{1}\left(\Sigma_{1}\right)$ and $f^{1} \in N(P)=N\left(E_{1}\right)$ by Theorem 2.1(i). It is obvious that $f_{1} \neq 0$. Then $P f_{1}=\lambda f_{1}+\lambda f^{1}$ which implies that

$$
P_{1} f_{1}=E_{1} P f_{1}=E_{1}\left(\lambda f_{1}+\lambda f^{1}\right)=\lambda f_{1} .
$$

Now let $f_{1}=f_{2}+f^{2}$ with $f_{2} \in L^{1}\left(\Sigma_{2}\right)$ and $f^{2} \in N\left(P_{1}\right)=N\left(\left.E_{2}\right|_{L^{1}\left(\Sigma_{1}\right)}\right)$. Then $f_{2} \neq 0$. Thus,

$$
P_{2} f_{2}=E_{2} P_{1} f_{2}=E_{2}\left(\lambda f_{2}+\lambda f^{2}\right)=\lambda f_{2} .
$$

Repeating the above process $P_{1}, P_{2}, \ldots, P_{k-1}$ successively, we have $P_{k} f_{k}=\lambda f_{k}$ for some nonzero $f_{k} \in L^{1}\left(\Sigma_{k}\right)$. Since $P_{k}$ is injective, Proposition 2.2 implies that $|\lambda|=1$.

More generally we have the following result. Suppose $\left(X, \Sigma_{\infty}, \mu\right)$ is $\sigma$-finite. Then $E_{\infty}$ and $E_{k}$ are well-defined for all $k$ 's. A lemma is given first.

Lemma 2.1. The operator $P_{i}$ maps $N\left(E_{k}\right)$ into itself for every $i$ and $k$ satisfying $0 \leq i \leq k$. Thus,

$$
E_{k} P_{i}=E_{k} P_{i} E_{k}=P_{k} E_{k}
$$

Proof. Let $f \in N\left(E_{k}\right)$. Then for any $A \in \Sigma_{k}$,

$$
\int_{A} E_{k} P_{i} f d \mu=\int_{A} P_{i} f d \mu=\int_{S^{-1}(A)} f d \mu=\int_{S^{-1}(A)} E_{k} f d \mu=0 .
$$

Hence, $E_{k} P_{i}=0$ on $N\left(E_{k}\right)$. Since $E_{k}$ is identity on $R\left(E_{k}\right)$ and $L^{1}\left(\Sigma_{i}\right)=R\left(E_{k}\right) \oplus$ $N\left(\left.E_{k}\right|_{L^{1}\left(\Sigma_{i}\right)}\right),(2)$ follows.

Proposition 2.4. If $E_{k} \rightarrow E_{\infty}$ strongly, then $\sigma_{p}(P) \subset \partial D \cap\{0\}$. 
Proof. Suppose $P f=\lambda f$ for some $\lambda \neq 0$ and $f \neq 0$ in $L^{1}$. Then $E_{k} P f=\lambda E_{k} f$. Letting $f_{k}=E_{k} f$ and using Lemma 2.1, we have

$$
P_{k} f_{k}=\lambda f_{k}, \quad k=1,2, \ldots
$$

Let $f_{\infty}=E_{\infty} f$. Then $\lim _{k \rightarrow \infty} f_{k}=f_{\infty}$. It follows that $\lim _{k \rightarrow \infty} P_{k} f_{k}=P_{\infty} f_{\infty}$ since from Lemma 2.1 ,

$$
\begin{aligned}
\left\|P_{k} f_{k}-P_{\infty} f_{\infty}\right\|_{1} & \leq\left\|P_{k} f_{k}-P_{k} f_{\infty}\right\|_{1}+\left\|P_{k} f_{\infty}-P_{\infty} f_{\infty}\right\|_{1} \\
& \leq\left\|f_{k}-f_{\infty}\right\|_{1}+\left\|E_{k} P f_{\infty}-E_{\infty} P f_{\infty}\right\|_{1} .
\end{aligned}
$$

Therefore, $P_{\infty} f_{\infty}=\lambda f_{\infty}$. The same argument as in the proof of Proposition 2.3 implies that $f_{\infty} \neq 0$. Since $S^{-1} \Sigma_{\infty}=\Sigma_{\infty}$, from Theorem 2.1(iii), $P_{\infty}$ is injective. Hence $|\lambda|=1$ by Proposition 2.2

Here is a sufficient condition for $\Sigma_{k+1}=\Sigma_{k}$. Let $h_{k}=d \mu \circ S^{-k} / d \mu$ and $\Sigma_{h_{k}}=$ $\left\{A \cap \operatorname{supp} h_{k}: A \in \Sigma\right\}$.

Proposition 2.5. If $\Sigma_{h_{k}} \subset \Sigma_{1}$, then $\Sigma_{k+1}=\Sigma_{k}$.

Proof. $S^{-k}\left(\operatorname{supp} h_{k}\right)=X$ since

$$
\mu\left(\left(S^{-k}\left(\operatorname{supp} h_{k}\right)\right)^{c}\right)=\mu\left(S^{-k}\left(\left(\operatorname{supp} h_{k}\right)^{c}\right)\right)=\int_{\left(\operatorname{supp} h_{k}\right)^{c}} h_{k} d \mu=0 .
$$

Given $B=S^{-k}(A)$ for some $A \in \Sigma$,

$$
B=S^{-k}\left(A \cap \operatorname{supp} h_{k}\right) \in S^{-k} \Sigma_{h_{k}} \subset S^{-k} \Sigma_{1}=\Sigma_{k+1} .
$$

Hence $\Sigma_{K+1}=\Sigma_{k}$.

\section{The Point SPECTRUM of $U$}

Now we study the point spectrum of the Koopman operator. Similar to Proposition 2.1, we have

Proposition 3.1. Suppose $S$ preserves $\mu$. If $U g=\lambda g$ with $\lambda \neq 1$ and $g \in L^{\infty} \cap L^{1}$, then $\int g d \mu=0$.

Proof. Since $\mu$ is $S$-invariant,

$$
\int g d \mu=\int U g d \mu=\lambda \int g d \mu
$$

which implies that $\int g d \mu=0$ since $\lambda \neq 1$.

Proposition 3.2. If $U$ is injective, then $\sigma_{p}(U) \subset \partial D$.

Proof. By Theorem 2.1(ii), $U$ is isometric. Hence

$$
0=\|U g-\lambda g\|_{\infty} \geq\|U g\|_{\infty}-|\lambda|\|g\|_{\infty}=(1-|\lambda|)\|g\|_{\infty} .
$$

Now $f \neq 0$ and $|\lambda| \leq 1$ imply that $|\lambda|=1$.

It is possible that $\sigma_{p}(U)=\partial D$ as the following examples show.

Example 3.1. Let $S:(-\infty, \infty) \rightarrow(-\infty, \infty)$ be defined by $S(x)=x+1$. Then the Lebesgue measure is invariant and $S^{-1} \Sigma=\Sigma$. Thus, $\sigma(U) \subset \partial D$ by Theorem 2.1(iii) and (vi). It is easy to see that $e^{i r}$ is an eigenvalue of $U$ with the eigenfunction $e^{i r x}$. Thus $\sigma(U)=\sigma_{p}(U)=\partial D$. 
The next example indicates that if $U$ is not injective, there may be eigenvalues $\lambda$ with $|\lambda|<1$.

Example 3.2. Let $S_{\alpha}:[0,1] \rightarrow[0,1]$ be defined by $S_{\alpha}(x)=\alpha x$ with $0<\alpha<1$. Then $U$ is not injective and $\sigma(U)=D$. For $g(x)=x^{n}$, we have

$$
U g(x)=(\alpha x)^{n}=\alpha^{n} x^{n}=\alpha^{n} g(x) .
$$

Hence $\left\{\alpha^{n}\right\}_{n=0}^{\infty} \subset \sigma_{p}(U)$. Since $\lim _{\alpha \rightarrow 1} S_{\alpha}=I$, the identity mapping of $[0,1]$, we see that not only the spectrum but also the point spectrum of $U$ is not continuous, but only upper semicontinuous.

Proposition 3.3. Suppose $S$ is ergodic and $U$ is injective. Then any unit eigenfunction $g$ of $U$ satisfies that $|g|=1$.

Proof. By Proposition 3.2, $|\lambda|=1$. Since

$$
U|g|=|U g|=|\lambda||g|=|g|,
$$

the ergodicity of $S$ implies that $|g|=1$.

Proposition 3.4. If $S$ is ergodic and $U$ is injective, then $\sigma_{p}(U)$ is a subgroup of $\partial D$ and each eigenvalue is simple.

Proof. Suppose $U g_{1}=\lambda_{1} g_{1}$ and $U g_{2}=\lambda_{2} g_{2}$ with $\left\|g_{1}\right\|_{\infty}=\left\|g_{2}\right\|_{\infty}=1$. By Proposition 3.2, $\left|\lambda_{1}\right|=\left|\lambda_{2}\right|=1$. From

$$
U\left(g_{1} g_{2}\right)=U g_{1} U g_{2}=\lambda_{1} g_{1} \lambda_{2} g_{2}=\lambda_{1} \lambda_{2} g_{1} g_{2}
$$

and $\left|g_{1} g_{2}\right|=\left|g_{1}\right|\left|g_{2}\right|=1$ by Proposition 3.3, $\lambda_{1} \lambda_{2} \in \sigma_{p}(U)$. Similarly, from

$$
U \frac{g_{1}}{g_{2}}=\frac{U g_{1}}{U g_{2}}=\frac{\lambda_{1} g_{1}}{\lambda_{2} g_{2}}=\frac{\lambda_{1}}{\lambda_{2}} \frac{g_{1}}{g_{2}}
$$

and $\left|g_{1} / g_{2}\right|=\left|g_{1}\right| /\left|g_{2}\right|=1$, we see that $\lambda_{1} / \lambda_{2} \in \sigma_{p}(U)$.

Let $g_{1}, g_{2}$ be eigenfunctions of $U$ with the same eigenvalue $\lambda$. Then

$$
U \frac{g_{1}}{g_{2}}=\frac{U g_{1}}{U g_{2}}=\frac{\lambda g_{1}}{\lambda g_{2}}=\frac{g_{1}}{g_{2}} \text {. }
$$

Since $S$ is ergodic, $g_{1} / g_{2}$ is a constant function. Hence $\lambda$ is simple.

Since $U$ is injective if $\mu$ is $S$-invariant [5], we immediately have the following corollary whose proof can be found in [17] when $U$ is unitary on $L^{2}$.

Corollary 3.1. If $S$ preserves $\mu$, then $\sigma_{p}(U) \subset \partial D$. If in addition $S$ is ergodic, then $\sigma_{p}(U)$ is a subgroup of $\partial D$ and each eigenvalue is simple with the corresponding unit eigenfunction $g$ satisfying $|g|=1$.

Finally, I thank the editor for help in extending the introduction.

\section{REFERENCES}

1. S. Albeverio and R. Hoegh-Krohn, Frobenius-theory for positive maps of von Neumann algebras, Comm. Math. Phys. 64 (1978), 83-94. MR 81m:46091

2. C. Beck and F. Schlögl, Thermodynamics of Chaotic Systems, Cambridge University Press, 1993. MR 94m:58134

3. C. Chiu, Q. Du and T.-Y. Li, Error estimates of the Markov finite approximation of the Frobenius-Perron operator, Nonlinear Anal. TMA 19 (4) (1992), 291-308. MR 93f:28014

4. J.Ding, Decomposition theorems for Koopman operators, Nonlinear Anal. TMA 28 (1997), 1011-1018. CMP 97:05 
5. J. Ding, Q. Du and T.-Y. Li, The spectral analysis of Frobenius-Perron operators, J. Math. Anal. Appl. 184 (2) (1994), 285-301. MR 95c:47007

6. J. Ding, Q. Du and T.-Y. Li, High order approximation of the Frobenius-Perron operator, Applied Math. Comput. 53 (1993), 151-171. MR 94d:65036

7. J. Ding and W. E. Hornor, A new approach to Frobenius-Perron operators, J. Math. Anal. Appl., 187 (3) (1994), 1047-1058. MR 95k:28033

8. J. Ding and T.-Y. Li, A convergence rate analysis for Markov finite approximations to a class of Frobenius-Perron operators, to appear: Nonlinear Anal. TMA.

9. N. Dunford and J. Schwartz, Linear Operators, Part I, General Theory, Interscience, 1958. MR 22:8302

10. D. E. Evans and R. Hoegh-Krohn, Spectral properties of positive maps on $C^{*}$-algebras, J. London Math. Soc. 17 (2) (1978), 345-355. MR 58:2319

11. M. Fannes, B. Nachtergaele, and R. F. Werner, Finitely correlated states on quantum spin chains, Comm. Math. Phys. 144 (1992), 443-490. MR 93i:82006

12. S. R. Foguel, The Ergodic Theory of Markov Processes, Van Nostrand Reinhold, 1969. MR 41:6299

13. F. Y. Hunt and W. M. Miller, On the approximation of invariant measures, J. Stat. Phys. 66 (1992), 535-548. MR 93a:58105

14. A. Lasota and M. Mackey, Chaos, Fractals, and Noises, Second Ed., Springer-Verlag, 1994. MR 94j:58102

15. T. Y. Li, Finite approximation for the Frobenius-Perron operator, a solution to Ulam's conjecture, J. Approx. Theory 17 (1976), 177-186. MR 54:811

16. H. H. Schaefer, Banach Lattices and Positive Operators, Springer-Verlag, 1974. MR 54:11023

17. Ya. G. Sinai, Topics in Ergodic Theory, Princeton University Press, 1994. MR 95j:28017

18. S. Ulam, A Collection of Mathematical Problems, Interscience, 1960. MR 22:10884

19. P. Walter, An introduction to Ergodic Theory, Springer-Verlag, 1982. MR 84e:28017

Department of Mathematics, The University of Southern Mississippi, Hattiesburg, MississipPI 39406-5045

E-mail address: jding@yizhi.st.usm.edu 